\title{
Advanced glycation end products and the pathogenesis of nonalcoholic fatty liver disease in non-diabetic adults
}

\author{
S.A. Palma-Duran ${ }^{1}$, M.D. Kontogianni ${ }^{2}$, A. Vlassopoulos ${ }^{1}$, S. Zhao ${ }^{1}$, G.V. Papatheodoridis ${ }^{3}$ and \\ E. Combet ${ }^{1}$ \\ ${ }^{1}$ Human Nutrition, School of Medicine, University of Glasgow, G31 2ER, UK, ${ }^{2}$ Department of Nutrition \& Dietetics, \\ School of Health Science and Education, Harokopio University, Kallithea, Greece and ${ }^{3} 2$ nd Department of Internal \\ Medicine, Athens University Medical School, Hippokration Hospital of Athens, Athens, Greece
}

Non-alcoholic fatty liver disease (NALFD) is considered as the hepatic manifestation of the metabolic syndrome, with insulin resistance (IR) as key underlying pathophysiological mechanism. NAFLD comprise a wide range of liver damage, from simple fatty liver to non-alcoholic steatohepatitis (NASH), including cirrhosis ${ }^{(1)}$. Hyperglycemia, IR and oxidative stress increase advance glycation end products (AGEs) that may contribute to the pathophysiology of NAFLD through the AGE-RAGE axis. AGEs such pentosidine, $\mathrm{N}^{\varepsilon}$-carboxyethyl-L-lysine (CEL) and $\mathrm{N}^{\varepsilon}$-carboxymethyl-L-lysine $(\mathrm{CML})$ exert pathological effects by binding to AGEs receptors (RAGE), triggering inflammation, cellular dysfunction and cell death. Soluble RAGE (sRAGE) may protect by preventing AGEs/ RAGE interaction ${ }^{(2)}$. The aim of the study was to evaluate the association of AGEs and sRAGE levels in a case (NAFLD)-control cohort with markers of liver function (ALT, AST, and GGT) and IR.

The study included 131 non-diabetic adults in age, sex and BMI matched pairs based on the presence/absence of NAFLD (liver enzymes and ultrasound hepatic steatosis) ${ }^{(3)}$. AGEs were analysed by liquid chromatography-mass spectrometry (CML, CEL), fluorescence (pentosidine, AGE fluorescence), colorimetry (fructosamine) and ELISA (sRAGE).

\begin{tabular}{|c|c|c|c|c|c|c|}
\hline & \multicolumn{2}{|c|}{ Cases $(n=73)$} & \multicolumn{2}{|c|}{ Controls $(n=58)$} & \multicolumn{2}{|c|}{ Total $(n=131)$} \\
\hline & Mean & SD & Mean & SD & Mean & SD \\
\hline$\overline{\operatorname{ALT}(\mathrm{IU} / \mathrm{L})^{\mathrm{a}}}$ & $77 \cdot 3^{*}$ & $47 \cdot 6$ & 14.9 & 5.9 & $50 \cdot 6$ & $\overline{47 \cdot 6}$ \\
\hline AST(IU/L) & $42 \cdot 3^{*}$ & $21 \cdot 1$ & $24 \cdot 6$ & $6 \cdot 7$ & $34 \cdot 8$ & $18 \cdot 7$ \\
\hline GGT $(\mathrm{IU} / \mathrm{L})^{\mathrm{a}}$ & $94 \cdot 1 *$ & $116 \cdot 3$ & $20 \cdot 1$ & $12 \cdot 0$ & $62 \cdot 3$ & $95 \cdot 3$ \\
\hline HOMA-IR ${ }^{\mathrm{a}}$ & $3 \cdot 6^{*}$ & $2 \cdot 5$ & 1.9 & 1.5 & $2 \cdot 8$ & $2 \cdot 3$ \\
\hline Fructosamine (mM DMFE) & $1 \cdot 12^{*}$ & $0 \cdot 2$ & 0.97 & $0 \cdot 96$ & 1.05 & $0 \cdot 2$ \\
\hline AGE fluorescence $(A U)^{\mathrm{a}}$ & $523 \cdot 5^{*}$ & $130 \cdot 9$ & $414 \cdot 3$ & $63 \cdot 88$ & $475 \cdot 1$ & $119 \cdot 3$ \\
\hline sRAGE (pg/L) & $397.9 *$ & 263.9 & $695 \cdot 1$ & 288.43 & $527 \cdot 5$ & $311 \cdot 1$ \\
\hline $\mathrm{CML}(\mathrm{mmol} / \mathrm{mol})$ & $10 \cdot 6$ & $3 \cdot 7$ & $9 \cdot 9$ & $9 \cdot 69$ & $1 \cdot 3$ & $3 \cdot 2$ \\
\hline CEL (mmol/mol) & $124 \cdot 0^{*}$ & $32 \cdot 5$ & $93 \cdot 4$ & $30 \cdot 81$ & $110 \cdot 2$ & $35 \cdot 1$ \\
\hline Pentosidine $(\mathrm{mmol} / \mathrm{mol})^{\mathrm{a}}$ & $1.7 *$ & $0 \cdot 4$ & 1.5 & $0 \cdot 8$ & 1.6 & $0 \cdot 3$ \\
\hline
\end{tabular}

Mean values of cases were significantly different from the controls (independent $t$-test, $* P<0.01$ selecting cases if match by age, sex and BMI). ${ }^{a}$ Data are not normally distributed.

Patients' mean age was 45 year with a BMI of $29 \mathrm{~kg} / \mathrm{m}^{2}$. Glycation biomarkers were significantly higher in cases compared to controls, except for CML ( $\mathrm{p}=0 \cdot 19)$. The absence of difference could be explained by the suppressed CML plasma levels observed in overweight subjects, thereby reducing our ability to detect an effect ${ }^{(4)}$. Compared to controls, AGEs were $36 \%$ higher in cirrhosis patients, $18 \%$ higher in NASH and $27 \%$ higher in fatty liver patients $(\mathrm{p}<0.01)$. Two-fold lower sRAGE levels were present in cases and were inversely associated with CEL and AGE fluorescence $(\mathrm{r}=0.59, \mathrm{p}<0 \cdot 01)$. sRAGE levels were inversely associated with the severity of NAFLD based on liver function and IR $(r=0.44, p<0.01)$, and the stage of the disease $(r=0.38, P<0.001)$. These findings support the hypothesis that AGEs-RAGE axis is associated with the reduction of hepatic function and highlight their potential as markers of NAFLD progression.

The cross-sectional study was financially supported by the Hellenic Foundation of Gastroenterology \& Nutrition. S.P acknowledges receipt of a CONACyT Ph.D. scholarship.

1. Ratziu V, Bellentani S, Cortez-Pinto H, et al. (2010). Journal of hepatology. 53, 372-384.

2. Yilmaz Y, Ulukaya E, Gul OO, et al. (2009). Clin Biochem. 42, 802-807.

3. Georgoulis M, Kontogianni MD, Tileli N, et al. (2014). Eur J Nutr. 53, 1727-1735.

4. Delgado-Andrade C (2016). Food Funct. 7, 46-57. 